\title{
Healthcare Design Metrics for Human-Centric Building Analytics
}

\author{
Khatereh Hadi \\ Georgia Tech Research Institute | USA | Khatereh.Hadi@gtri.gatech.edu \\ Paula Gomez \\ Georgia Tech Research Institute | USA | Paula.Gomez@gtri.gatech.edu \\ Matthew Swarts \\ Georgia Tech Research Institute | USA | Matthew.Swarts@gtri.gatech.edu \\ Tyrone Marshall \\ Design Process Lab, Perkins\&Will | USA | Tyrone.Marshall@perkinswill.com \\ Marcelo Bernal \\ Director of Design Process Lab, Perkins\&Will | USA | Marcelo.Bernal@perkinswill.com
}

\begin{abstract}
Healthcare design practice has shown increasing interest in the assessment of design alternatives from a human-centered approach, focusing on organizational performance, patient health, and wellness outcomes, in addition to building performance. The goal of this research is to advance building analytics by identifying, defining and implementing computational human-centered design metrics. The knowledge is extracted from an exhaustive literature review in the field of evidence-based design (EBD), which has studied the associations between building features and the occupants' outcomes but has not yet consolidated the findings into metrics and implications for design practice in a systematic manner. In consultation with industry experts, we have prioritized the evaluation aspects and developed a weighted evaluation framework for assessment of various design options. The developed metrics that input building parameters and output potential health and performance outcomes are implemented in a a parametric environment utilizing add-ons accordingly, and using an ambulatory clinic designed by Perkins\&Will as a case study.
\end{abstract}

Keywords: Building analytics; Healthcare design; Design metrics; Human-centered analytics

\section{INTRODUCTION}

The goal of this research is to identify, define and integrate human-centered healthcare design metrics to extend building analytics. This paper presents a methodology to integrate evidence-based design (EBD) knowledge on healthcare design, extracted from a literature review, into a set of human-centered design metrics based on specific parameters of the building. These parameters will be later used to evaluate design options against these metrics for improving human performance outcomes. Numerous studies have highlighted design strategies that can improve health and wellness measures in healthcare environments. However, these research findings are dispersed and have limited implications in design practice due to the lack of unified, quantifiable metrics for measuring both building and associated human-centered outcomes, in this case, health and performance outcomes. In the presented research, we addressed this issue by understanding the relationships between building design parameters and reported health and wellness outcomes, presenting a framework for healthcare design evaluation based on these findings.

\section{METHODS}

The four stages of this research are knowledge collection, prioritization of metrics, proposed methods for implementation of the selected metrics as computational parameters, and their implementation in computational models. The body of knowledge acquired through research in healthcare design and operation is consolidated through the following stages:

a. Exploratory review of related literature.

b. Identification of human-design correlations and quantifiable design metrics, along with their associated performance, and health and wellness outcomes.

c. Prioritization of metrics in consultation with industry partners.

d. Development of assessment algorithms for analyzing buildings' health impacts based on the defined metrics.

\section{LITERATURE REVIEW}

We first conducted a literature review, looking for the existing research studies highlighting the relations between healthcare design and patient/staff outcomes to identify design configurations and metrics that have been repeatedly studied by healthcare design researchers. We used a snowball method to locate and retrieve studies to include in our literature review. Our literature review included searching academic databases and leading 
health design journals and magazines including Health Environments Research \& Design (HERD), Environment and Behavior, Journal of Environmental Psychology, nursing journals, AIA (American Institute of Architects) Academy Journal, the Center for Health Design reports, white papers and conference proceedings. A total of 74 articles were included in this review.

\section{IDENTIFY METRICS}

Through a preliminary review of our literature search, we compiled a comprehensive taxonomy of healthcare design features related to patient and staff outcomes in healthcare environments. These design features included walking distances, circulations, accessibility, patient visibility, lighting, outside views, flexibility, layout types, nurse station typologies, patient room typologies, unit size, acoustics, density /crowding, and thermal comfort.

We conducted an in-depth literature review and analysis on each design feature. For each design feature, we compiled a list of studies with relevant design metrics, the operationalized definition of each design metrics, the outcome metrics, and the associations between the identified design metrics and the outcomes metrics (percentage or amount of improvement or deterioration in outcomes related to each design metric). We then compared and summarized research findings for similar metrics across multiple studies.

\section{PRIORITIZE DESIGN FEATURES}

Through first rounds of meetings with the healthcare design experts from our partner architecture company Perkins\&Will, four design features were identified as priorities for in-depth research based on the experience of the experts interacting with clients: 1) walking distances, 2) patient visibility, 3) light, and 4) outside views. In the second round of meetings, three additional design features were added to the research priority list, adding up to 7 priorities: 5) flexibility 6) unit size, and 7) patient room typologies. We identified several parameters and metrics utilized to measure each of these seven design features across different studies, associated with a variety of different outcomes. In order to select the design metrics for our assessment, we consulted with Perkins\&Will healthcare design experts to prioritize the associated outcomes in terms of the client and project goals. Through this exercise, we prioritized the outcomes and identified the design metrics utilized to measure those outcomes. It is important to mention that this research was performed before and during the COVID-19 pandemic, and the priority of some outcomes changed due to the pandemic response needs (marked in orange in table 1).

Table 1: Matrix describing the design features with their associated metrics, and the human-centric performance outcomes impacted. The blue cells show the outcomes prioritized by experts, and the red cells show the outcome affected by COVID-19 (family presence), which changed from having a positive impact to a negative impact on health.

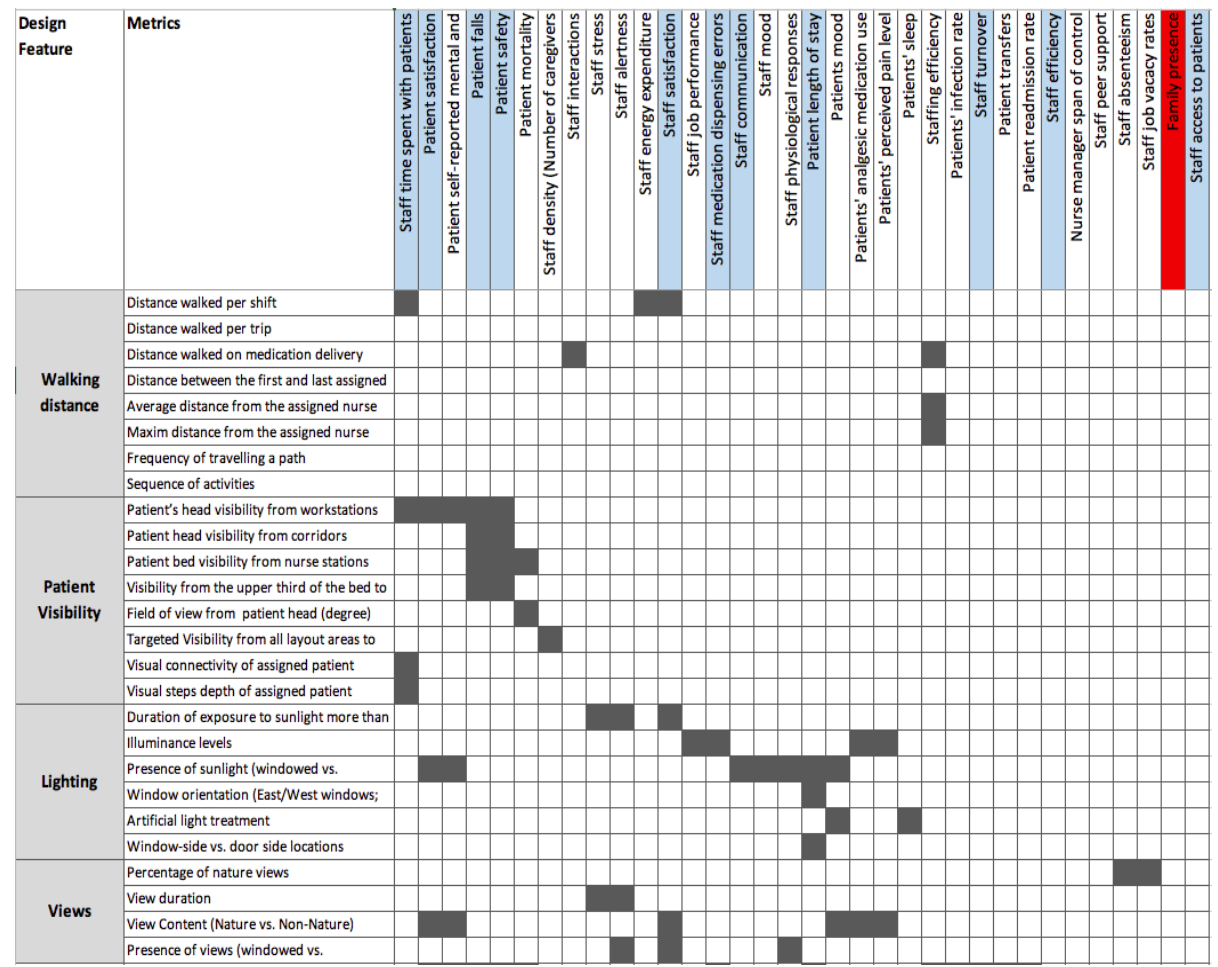


Table 2: Continuation of Table 1, representing the outcomes of the second meeting (Wil be included in future publications).

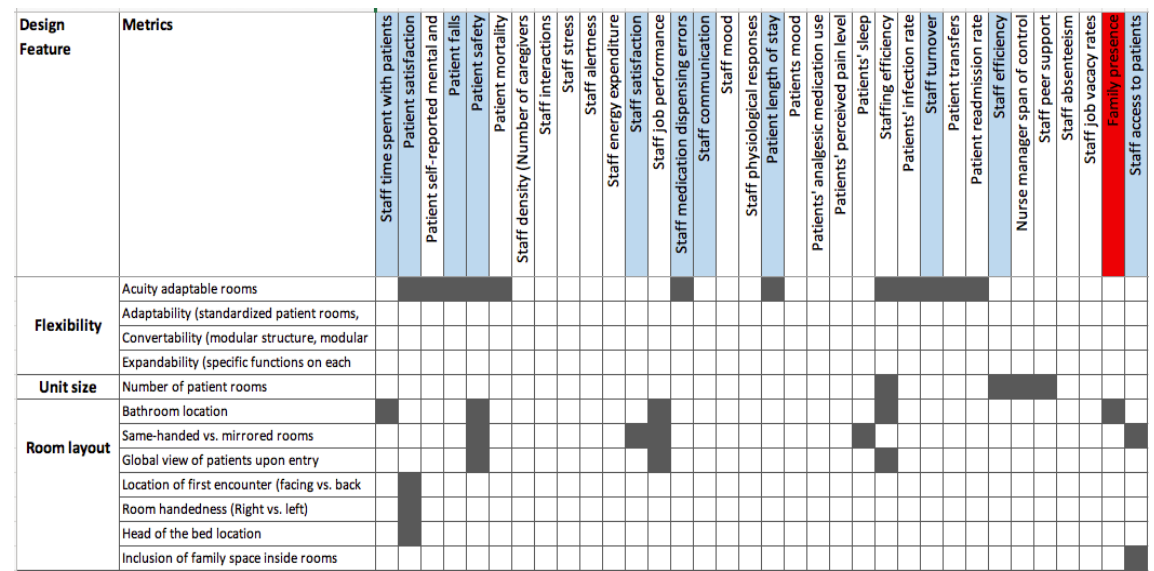

DESIGN METRICS AND

\section{COMPUTATIONAL ASSESSMENT}

In this section, we present definitions, metrics and computational methods for measuring the first four of the seven aforementioned design features: Walking distances, patient visibility, lighting, and outside views. The rest of the features directly associated with the layout configuration are currently being studied as this research is in progress, and they will be presented in a follow-up publication.

\section{WALKING DISTANCES}

Although several researchers have studied this topic, there is no universal walking distance thresholds for designing patient units. The distance travelled per-shift in studies included in this review ranged between 1 to 6.8 miles (1.6 to $10.9 \mathrm{Km})$. The design and operations of different patient units can impact the nurses' walking distances through parameters such as layout typologies, number of patient assignments, frequency of visiting different locations, and sequence of visiting different location for each task.

The metrics to measure the nurses' walking distances reported in the literature included: distance walked per shift (Copeland \& Chambers ,2017; Elganzouri, et al., 2009; Hendrich, et al., 2008; Nanda, et al 2015; Pati, et al , 2015; Shepley \& Davies, 2003; Welton, et al., 2006), per specific care processes (Elganzouri, et al., 2009; Seo, et al., 2011; Yi \& Seo, 2012) and per specific destination in the unit (Acar \& Butt, 2016; Copeland \& Chambers, 2017; Hendrich et al., 2009; Nanda et al., 2015; Real et al., 2017; Welton, et al., 2006). Based on the literature review, we identified the below metrics for calculating the walking distances (from/to) for patient units per shift:
a. Patients' room to medication rooms/stations
b. Patients' room to patients' room
c. Patients' room - utility/supplies rooms
d. Patients' room - stations
e. Stations - stations,
f. Stations - utility/supplies rooms
g. Medication rooms - medication rooms
h. Medication rooms - utility/supplies rooms
i. Utility/supplies rooms - utility/supplies rooms.

For the implementation of walking distances, we relied on a pathfinding algorithm based on line of sights within a navigation map that represents the walkable spaces as a two-dimensional grid of points (Naderpour, Johnson \& Anderson, 2019). Unlike other algorithms that zigzag through the grid, the adopted algorithm connects points based on visibility that represent behavior of people walking through the space. This visual navigation approaches produces more accurate trajectories and lengths of the routes. The representation of the routes as polylines allows the quantification of individual and global walking distances and estimated travel time (Figure 1). These routes require origin and destination points, either one-to-one one-to-many, which facilitates the specialization of any scheduled activity. In addition, a macro grid keeps track the number of routes going across each macro cell to report the potential congested areas (Figure 2).

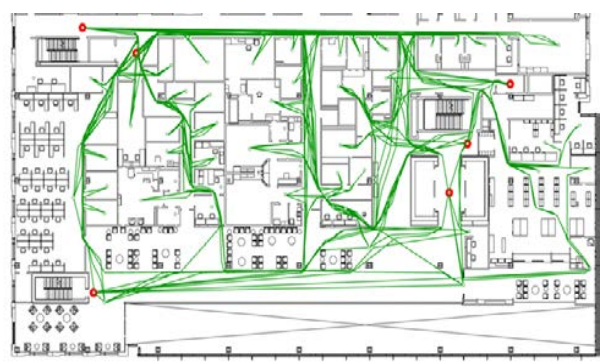

Figure 1: All possible routes from every room to reach the elevators and stairs labeled with a red point 


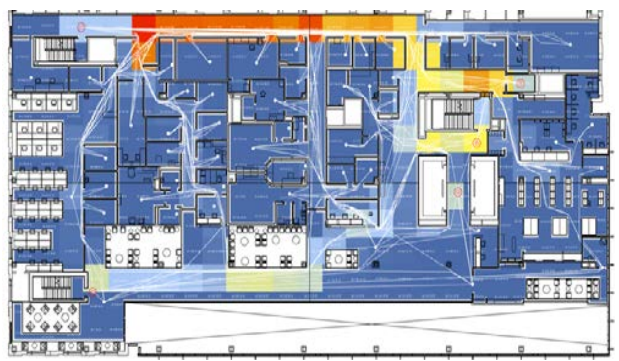

Figure 2: Macro-grid mapping potential congested areas

\section{PATIENT VISIBILITY}

The existing studies on the design of inpatient units show how visibility to patients from nurses' work areas is associated with patient outcomes including patient falls (Bosch et al., 2016; Calkins et al., 2012; Choi, 2011; Vassallo et al., 2000), mortality rates (Leaf et al., 2010; Lu et al., 2014) and patient satisfaction (Bosch et al., 2016). Patient visibility from nurse stations, staff work areas, and corridors impacts opportunities for direct patient care and observation (Bosch et al., 2016; Heo et al., 2009; Lu \& Zimring, 2012).

Although patient visibility is usually used as a general term, various metrics have been defined to measure it across different studies including patient head visibility from nurse stations and corridors (Bosch et al., 2016; Choi, 2011), patient bed visibility from nurse stations (Leaf et al., 2010; Vassallo et al., 2000), visibility of upper third part of the bed from nurse stations (Calkins et al., 2012), field of view from patient head (Lu et al., 2014), patient bed visibility across the entire unit (targeted visibility) (Lu \& Zimring, 2012), visual connectivity of patient rooms, visual step depth of assigned patient rooms (Heo et al., 2009), and Isovistminute, a spatiotemporal metric that measures real surveillance by including the actual occupancy of the space (Gomez-Z., P., 2017; Gomez-Z. P. et al, 2019). Based on the literature review and prioritization of metrics with healthcare design experts, patients' visibility and visual connectivity of patient rooms were selected as the visibility metrics to implement in the calculation algorithm.

The methods to measure the variety of metrics for patients head visibility from different locations and perspectives are based on variations of implementations of the Isovist (Benedict, 1979). The Isovist algorithm defines a visibility plane that represents the field of view from the location of the human eye, mapping the obstacles obstructing the view, and quantifies the visible area (Figure 3). If we apply this algorithm across all points in a layout, we can evaluate the degree of the visibility of a specific location to all other locations (Visual Connectivity or Visibility Area). While the evaluation of the visibility of a particular target, such as a patient's head, allows us to verify the visibility from specific location such as a nurse station to ensure patient observation and safety, the visual connectivity evaluates the visual connection across the space which has been shown to be related to team communication and security. The toolkit DecodingSpaces, implemented by a community of global collaborators, supports a variety to Isovist-based metrics developed in the field of Space Syntax (Abdulmalik Abdulmawla et al., 2019).
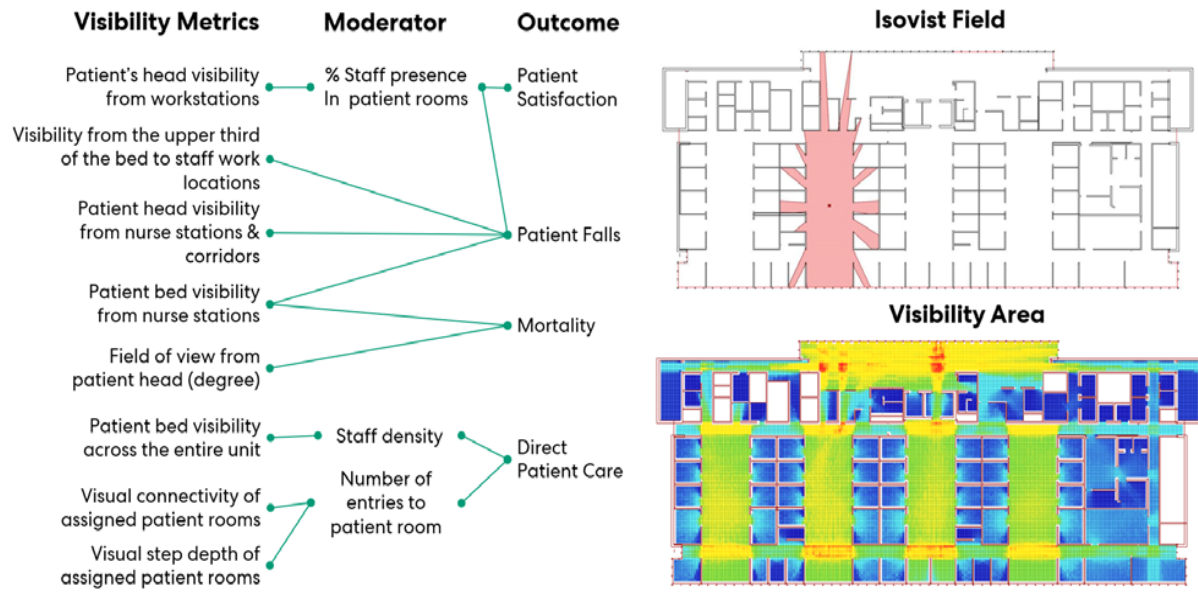

Figure 3: a) Visibility Metrics and their impact on human responses (outcomes); b) Isovist and Visual Connectivity (or Visibility Area by DecodingSpaces toolkit).

\section{LIGHTING}

The impact of exposure to light, specifically sunlight, has been studied in relation to both patients and staff outcomes in healthcare environments. Exposure to light can be associated with staff satisfaction, stress (Alimoglu \& Donmez, 2005), mood, communications, physiological responses (Zadeh et 
al., 2014), visual task performance (Figueiro et al., 2006), and medication dispensing errors (Buchanan et al., 1991). The existing studies also show the relationship between light exposure and patient outcomes including patient satisfaction (Gharaveis et al., 2016), length of stay (Beauchemin \& Hays, 1996; Benedetti et al., 2001; Choi et al., 2012; Park et al., 2018), mood (Beauchemin \& Hays, 1996; Walch et al., 2005), pain (Walch et al., 2005; Zadeh et al., 2014), and sleep (Hadi et al., 2019). Within the context of these studies, lighting has been measured through a variety of metrics such as:

a. The presence of sunlight (windowed vs. windowless locations).

b. Illuminance levels

c. Duration of exposure to light

d. Timing of exposure to light (morning, mid-day, evening)

e. Distance to light source (window-side vs. door-side locations)

Based on prioritization of metrics, we selected duration of exposure and illuminance levels as the lighting metrics to implement in calculations. We used ClimateStudio tool to measure Spatial Daylight Autonomy (sDA), which is the percentage of the floor area that receive illuminance levels within 300-3000 lux for at least $50 \%$ of the annual occupied hours, according to LEED V4 (Figure 4), duration and timing of daylight exposure (Figure 5) and distance to light sources in different areas of the building. ClimateStudio (Solemma, 2020), is a dynamic daylight simulation tool that supports several daylight metrics and reports annual and point-in-time results. It provides access and visualization of illuminance over time. This feature allows overlapping the duration of exposure to the target daylight thresholds in each space and the scheduled activities in the same area.

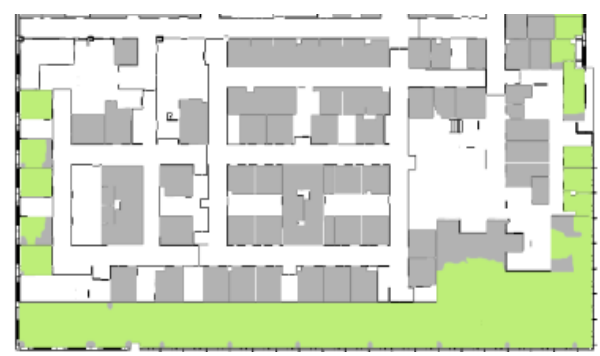

Figure 4: Mapping of Spatial Daylight Autonomy (SDA).

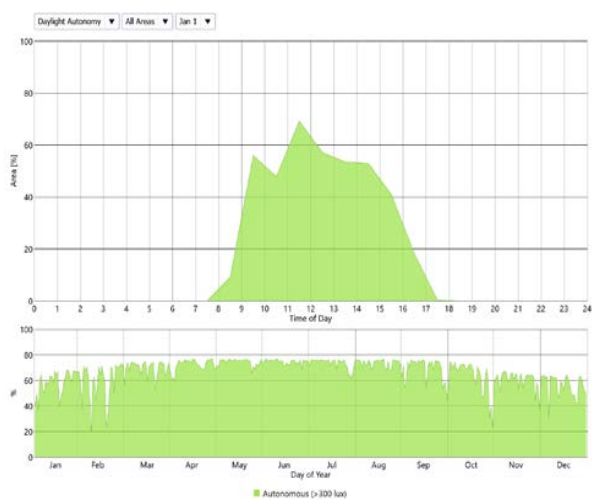

Figure 5: Visualization of daylight intensity along the day in the area of interest.

\section{ACCESS TO VIEWS}

The impact of access to windows on patients and staff has been studied from two aspects: access to sunlight and access to outside views through windows. The existing studies show that access to outside views has been associated with staff outcomes such as absenteeism, job vacancy rates (Shepley et al., 2012), alertness, stress levels (Pati, Harvey Jr, et al., 2008), satisfaction (Nejati et al., 2016), physiological responses and self-reported sleepiness (Zadeh et al., 2014). It has been also associated with patients' outcomes such as mood (Ulrich, 1984), pain (Ulrich, 1984; Wang et al., 2019a), satisfaction (Kearney \& Winterbottom, 2005), and self-reported mental health and physical health (Raanaas et al., 2012). Several metrics are used to measure access to outside views including assignment to windowed versus windowless locations, view content (nature versus nonnature), percentage of nature views, and the duration of exposure to outside views. The first two metrics were prioritized to be included in the calculation algorithms.

To ensure the visual connection with the surrounding landscape design and nature, we need to verify the access to views of the outdoors. In this regard, LEED V4 provides metrics to measure the quality of views (LEED, 2019). We created a grid of points at the human eye level in a working position at 1.2 meter from the floor, and implemented recommend metrics to measure the visual connection with the landscape: Lines of sight via vision glazing for $75 \%$ of all regularly occupied floor area, multiple lines of sight to vision glazing in different directions at least 90 degrees apart, and direct line of sight from three times the window head height. An additional raytracing method was utilized to quantify the visibility of specific targets such as (1) flora, fauna, or sky; (2) movement; and (3) objects at least 25 feet from the exterior of the glazing.

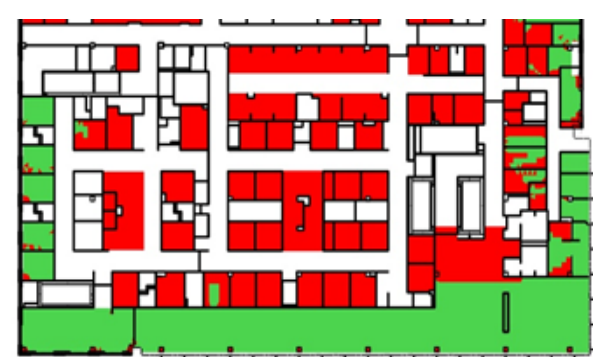

Figure 6: Occupied areas with line of sight to outdoors (green) and without line of sight to outdoors (red).

\section{DISCUSSION}

In recent years, we have witnessed advances in both fields, evidence-based design (EBD) studies for human-centric outcomes, and computational design evaluation methods for building-centric analytics. While EBD research studies show how design features, such as visibility and walking distances, are associated with different human outcomes, computational design research has developed various workflows to analyze and assess the value of the architectural layouts based on these features. Our 
research presents an approach for connecting both fields by assessing the computational building analytics results from the perspective of human outcomes.

The presented research is a collaborative effort by a team of GTRI healthcare design analytics researchers and expert healthcare designers and computational designers from Perkins\&Will practice and research, to help bridge this gap and move towards a computational design approach that includes existing evidence and expert knowledge towards human-centric healthcare outcomes such as stress levels, patient satisfaction, mortality rates, and patient care, among several others.

The workflow is based on existing computational methods for building analytics, but the assessments of the results are based on research and expert knowledge in a specific field. The area of building analytics sometimes presents conflicting objectives. Patient bathroom location and design is an example of such tradeoffs. While in-board bathroom locations offer optimum light and views for patients, they limit nurses' visibility to patients and increase travel distance to patient beds. Outboard bathrooms, on the other hand, optimize nurses' access and visibility to patient but limit natural views and lighting for patients. Other parameters are directly correlated to the objectives. For example, when Illuminance level increases, health outcomes such as stress levels, staff and patients' satisfaction, staff's task performance and patient's length of stay outcomes improve. When access to outdoor views increase, health outcomes such as stress levels, mood, and pain decrease.

Therefore, by using multi-objective computational optimization frameworks, implementing research-based scoring features and integrating customizable, projectbased weighting system based on expert knowledge, we can further advance building analytics to include potential human outcomes as a measure of building performance. This research extends the Design Space Construction (DSC) approach proposed by Haymaker et.al (2018), in which they "explore the design space maximizing social, environmental and economic value." This added layer of health and performance human-centered metrics utilizes the building as the static platform for building analytics, providing meaning to the outcomes related to patients and staff feedback. Such health, building-related metrics are activated depending on the organization operations. And, although various computational methods have been developed to analyze building layouts against standards, the thresholds recommended by current standards are not in alignment with the metrics recommended by the current research in areas such as walking distances, daylight levels, access to views and interior visibility.

Even though the implementation methods presented in this article could be generalizable to all healthcare building typologies, the proposed human-centered metrics are specific to ambulatory clinics. The values of these metrics depend on the specific goals, which in turn are tailored by the building organizational and operational targets. Some of these models and metrics are expected to be validated in Post-Occupancy Evaluations (POE) as a long-term research, including tracking methods for some of the metrics (i.e. walking distances), and surveys for others (i.e. level of stress).
Other challenges include spatiotemporal parameters. For example, the building analytics indicates the range of daylight on surfaces. However, from a human-centric perspective, it should also include the actual duration of exposure of occupants to daylight. Future work includes adding spatiotemporal parameters to the model, to expand it from a theoretical use of the space to the actual occupancy and activities performed. These parameters, combined with surveys, will help validate the health and performance outcomes that have been well studied in literature, but not implemented into building analytics until now.

\section{ACKNOWLEDGEMENTS}

This is a research collaboration between the Georgia Tech Research Institute and Perkins\&Will, funded by the state of Georgia and Perkins\&Will. We thank all internal advisors and experts in Healthcare design for their valuable feedback: Marvina Williams, Bill Nation. Diana Davis, and Amy Sickeler.

\section{REFERENCES}

Abdulmalik Abdulmawla, Bielik, M., Dennemark, M., Fuchkina, E., Miao, Y., Knecht, K., . . . Buš, P. (2019). DecodingSpaces Toolbox. Retrieved from https://toolbox.decodingspaces.net/\#aboutToolbox

Acar, I., \& Butt, S. E. (2016). Modeling nurse-patient assignments considering patient acuity and travel distance metrics. Journal of Biomedical Informatics, 64, 192-206.

Alimoglu, M. K., \& Donmez, L. (2005). Daylight exposure and the other predictors of burnout among nurses in a University Hospital. International Journal of Nursing Studies, 42(5), 549555.

Astley, P., Capolongo, S., Gola, M., \& Tartaglia, A. (2015). Operative and design adaptability in healthcare facilities. Techne-Journal of Technology for Architecture and Environment, 162-170.

Beauchemin, K. M., \& Hays, P. (1996). Sunny hospital rooms expedite recovery from severe and refractory depressions. Journal of Affective Disorders, 40(1-2), 49-51.

Benedetti, F., Colombo, C., Barbini, B., Campori, E., \& Smeraldi, E. (2001). Morning sunlight reduces length of hospitalization in bipolar depression. Journal of Affective Disorders, 62(3), 221223.

Benedikt, M. L. (1979). To take hold of space: isovists and isovist fields. Environment and Planning B: Planning and design, 6(1), 47-65.

Bosch, S. J., Apple, M., Hiltonen, B., Worden, E., Lu, Y., Nanda, U., \& Kim, D. (2016). To see or not to see: Investigating the links between patient visibility and potential moderators affecting the patient experience. Journal of Environmental Psychology, 47, 33-43

Buchanan, T. L., Barker, K. N., Gibson, J. T., Jiang, B. C., \& Pearson, R. E. (1991). Illumination and errors in dispensing. American Journal of Hospital Pharmacy, 48(10), 2137-2145. https://doi.org/10.1093/ajhp/48.10.2137

Buffoli, M., Nachiero, D., \& Capolongo, S. (2012). Flexible healthcare structures: analysis and evaluation of possible strategies and technologies. Ann lg, 24(6), 543-552.

Calkins, M. P., Biddle, S., Biesan, O., \& Institute, I. (2012). Contribution of the designed environment to fall risk in hospitals.

Catrambone, C., Johnson, M. E., Mion, L. C., \& Minnick, A. F. (2009). The design of adult acute care units in U.S. 
hospitals. Journal of Nursing Scholarship, 41(1), 79-86. https://doi.org/10.1111/j.1547-5069.2009.01254.x

Choi, J.-H., Beltran, L. O., \& Kim, H.-S. (2012). Impacts of indoo daylight environments on patient average length of stay (ALOS) in a healthcare facility. Building and Environment, 50, 65-75.

Choi, Y.-S. (2011). The physical environment and patient safety an investigation of physical environmental factors associated with patient falls. Thesis (Ph.D)--Architecture, Georgia Institute of Technology, 2012.

Choi, Y.-S., \& Bosch, S. J. (2013). Environmental Affordances: Designing for Family Presence and Involvement in Patien Care. HERD: Health Environments Research \& Design Journal, 6(4),

https://doi.org/10.1177/193758671300600404

Copeland, D., \& Chambers, M. (2017). Effects of unit design on acute care nurses' walking distances, energy expenditure, and job satisfaction: A pre-post relocation study. HERD: Health Environments Research \& Design Journal, 10(4), 22-36.

Cornell, P., Herrin-Griffith, D., Keim, C., Petschonek, S., Sanders, A. M., D'mello, S., Golden, T. W., \& Shepherd, G. (2010). Transforming nursing workflow, part 1: the chaotic nature of nurse activities. JONA: The Journal of Nursing Administration, 40(9), 366-373.

Elganzouri, E., Standish, C., \& Androwich, I. M. (2009). The mat study: global insight into the medication administration process. Nursing Informatics, 424-428.

Figueiro, M. G., Appleman, K., Bullough, J. D., \& Rea, M. S (2006). A discussion of recommended standards for lighting in the newborn intensive care unit. Journal of Perinatology, 26(S3), S19.

Gharaveis, A., Shepley, M. M., \& Gaines, K. (2016). The role of daylighting in skilled nursing short-term rehabilitation facilities. HERD: Health Environments Research \& Design Journal, 9(2), 105-118.

Gomez-Zamora, P. (2017) SPATIOTEMPORAL OCCUPANCY IN BUILDING SETTINGS, Ph.D. Thesis, Georgia Institute of Technology

Gomez-Zamora, P., Bafna, S., Zimring, C., Do, E., \& Romero Vega, M. (2019). Spatiotemporal Occupancy for Building Analytics.

Hadi, K., Du Bose, J. R., \& Choi, Y.-S. (2019). The Effect of Light on Sleep and Sleep-Related Physiological Factors Among Patients in Healthcare Facilities: A Systematic Review. HERD Health Environments Research \& Design Journal, 12(4), 116141.

Haymaker, J., Bernal, M., Marshall, M. T., Okhoya, V., Szilasi, A., Rezaee, R., ... \& Hasan, H. (2018). Design space construction: a framework to support collaborative, parametric decision making. Journal of Information Technology in Construction, 157178.

Hendrich, A., Chow, M. P., Bafna, S., Choudhary, R., Heo, Y., \& Skierczynski, B. A. (2009). Unit-related factors that affect nursing time with patients: spatial analysis of the time and motion study. HERD, 2(2), 5.

Hendrich, A., Chow, M. P., Skierczynski, B. A., \& Lu, Z. (2008). A 36-hospital time and motion study: how do medical-surgical nurses spend their time? The Permanente Journal, 12(3), 25.

Heo, Y., Choudhary, R., Bafna, S., Hendrich, A., \& Chow, M. P. (2009). A modeling approach for estimating the impact of spatia configuration on nurses' movement. Proceedings of the 7 th International Space Syntax Symposium, 41

Kearney, A. R., \& Winterbottom, D. (2005). Nearby nature and long-term care facility residents: Benefits and design recommendations. Journal of Housing for the Elderly, 19(3-4), 728. https://doi.org/10.1300/J081v19n03 02

Lavender, S. A., Sommerich, C. M., Patterson, E. S., Sanders, E. B. N., Evans, K. D., Park, S., Umar, R. Z. R., \& Li, J. (2015) Hospital patient room design: The issues facing 23 occupational groups who work in medical/surgical patient rooms. Health Environments Research and Design Journal, 8(4), 98-114. https://doi.org/10.1177/1937586715586391

Leaf, D. E., Homel, P. \& Factor, P. H. (2010). Relationship between ICU design and mortality. Chest, 137(5), 10221027.

LEED. (2019). View Quality. Retrieved from https://www.usgbc.org/credits/new-construction-commercialinteriors-schools-new-construction-retail-new-constructionret-2

Lovell, B. B., Ancoli-Israel, S., \& Gevirtz, R. (1995). Effect of bright light treatment on agitated behavior in institutionalized elderly subjects. Psychiatry Research, 57(1), 7-12.

Lu, Y., Ossmann, M. M., Leaf, D. E., \& Factor, P. H. (2014). Patient visibility and ICU mortality: A conceptual replication. HERD: Health Environments Research \& Design Journal, 7(2), 92103.

Lu, Y., Peponis, J., \& Zimring, C. (2009). Targeted visibility analysis in buildings: Correlating targeted visibility analysis with distribution of people and their interactions within an intensive care unit. In International Space Syntax Symposium.

Lu, Y., \& Zimring, C. (2012). Can Intensive Care Staff See Their Patients? An Improved Visibility Analysis Methodology. Environment and Behavior, 44(6), 861-876. https://doi.org/10.1177/0013916511405314

MacAllister, L. (2014). The impact of spatial layouts on patient satisfaction. In Unpublished manuscript, Georgia Institute of Technology, Atlanta, GA: College of Architecture.

Maze, C. (2009). Inboard, outboard, or nested? Healthcare Design Magazine.

Naderpour, A., Johnson, B. R., \& Anderson, A. (2019). A2B: A Toolkit for Computing Circulation Metrics in Buildings. Pape presented at the 16th Conference of the International Building Performance Simulation Association, IBPSA Rome.

Nanda, U., Pati, S., \& Nejati, A. (2015). Field research and parametric analysis in a medical-surgical unit. HERD: Health Environments Research \& Design Journal, 8(4), 41-57.

Nazarian, M., Price, A., Demian, P., \& Malekzadeh, M. (2018) Design lessons from the analysis of nurse journeys in a hospital ward. HERD: Health Environments Research \& Design Journal, 11(4), 116-129.

Nejati, A., Shepley, M., Rodiek, S., Lee, C., \& Varni, J. (2016). Restorative Design Features for Hospital Staff Break Areas: A Multi-Method Study. Health Environments Research and Design Journal, 9(2), 16-35. https://doi.org/10.1177/1937586715592632

Park, M. Y., Chai, C.-G., Lee, H.-K., Moon, H., \& Noh, J. S. (2018). The effects of natural daylight on length of hospital stay. Environmental Health Insights, 12, 1178630218812817.

Pati, D., Cason, C., Harvey Jr, T. E., \& Evans, J. (2010). An empirical examination of patient room handedness in acute medical-surgical settings. HERD: Health Environments Research \& Design Journal, 4(1), 11-33.

Pati, D., Harvey Jr, T. E., \& Barach, P. (2008). Relationships between exterior views and nurse stress: An exploratory examination. HERD: Health Environments Research \& Design Journal, 1(2), 27-38.

Pati, D., Harvey Jr, T. E., Redden, P., Summers, B., \& Pati, S. (2015). An empirical examination of the impacts of decentralized nursing unit design. HERD: Health Environments Research \& Design Journal, 8(2), 56-70.

Pati, D., Harvey Jr, T. E., Reyers, E., Evans, J., Waggener, L., Serrano, M., Saucier, R., \& Nagle, T. (2009). A multidimensional framework for assessing patient room configurations. HERD: Health Environments Research \& Design Journal, 2(2), 88-111. 
Raanaas, R. K., Patil, G. G., \& Hartig, T. (2012). Health benefits of a view of nature through the window: A quasi-experimental study of patients in a residential rehabilitation center. Clinical Rehabilitation, 26(1),21-32.

Rashid, M. (2014). Space Allocation in the Award-Winning Adult ICUs of the Last Two Decades (1993-2012): An Exploratory Study. HERD: Health Environments Research \& Design Journal, 7(2), 29-56. https://doi.org/10.1177/193758671400700203

Real, K., Bardach, S. H., \& Bardach, D. R. (2017). The role of the built environment: How decentralized nurse stations shape communication, patient care processes, and patient outcomes. Health Communication, 32(12), 1557-1570.

Shepley, Mardelle McCuskey, Gerbi, R. P., Watson, A. E., Imgrund, S., \& Sagha-Zadeh, R. (2012). The impact of daylight and views on ICU patients and staff. HERD: Health Environments Research \& Design Journal, 5(2), 46-60.

Silvis, J. (2011). Applying Evidence-Based Design at the New Parkland Hospital. Healthcare Design Magazine.

Smonig, R., Magalhaes, E., Bouadma, L., Andremont, O., de Montmollin, E., Essardy, F., Mourvillier, B., Lebut, J., Dupuis, C., \& Neuville, M. (2019). Impact of natural light exposure on delirium burden in adult patients receiving invasive mechanical ventilation in the ICU: a prospective study. Annals of Intensive Care, 9(1), 120.

The Advisory Board. (2007). Hospital of the future: Lessons for inpatient facility planning and strategy. https://www.advisory.com/research/health-care-advisoryboard/studies/2007/hospital-of-the-future

Thompson, D. R., Hamilton, D. K., Cadenhead, C. D., Swoboda, S. M., Schwindel, S. M., Anderson, D. C., Schmitz, E. V., St. Andre, A. C., Axon, D. C., Harrell, J. W., Harvey, M. A., Howard, A., Kaufman, D. C., \& Petersen, C. (2012). Guidelines for intensive care unit design*. Critical Care Medicine, 40(5), 1586-1600.

https://doi.org/10.1097/CCM.0b013e3182413bb2

Ulrich, R. (1984). View through a window may influence recovery. Science, 224(4647), 224-225.

Ulrich, R. S., Bogren, L., Gardiner, S. K., \& Lundin, S. (2018). Psychiatric ward design can reduce aggressive behavior. Journal of Environmental Psychology, 57, 53-66.

Vassallo, M., Azeem, T., Pirwani, M. F., Sharma, J. C., \& Allen, S. C. (2000). An epidemiological study of falls on integrated general medical wards. International Journal of Clinical Practice, 54(10), 654-657.
Walch, J. M., Rabin, B. S., Day, R., Williams, J. N., Choi, K., \& Kang, J. D. (2005). The effect of sunlight on postoperative analgesic medication use: a prospective study of patients undergoing spinal surgery. Psychosomatic Medicine, 67(1), 156-163.

Wang, C.-H., Kuo, N.-W., \& Anthony, K. (2019a). Impact of window views on recovery-an example of post-cesarean section women. International Journal for Quality in Health Care, 31(10), https://doi.org/10.1093/intqhc/mzz046

Wang, C.-H., Kuo, N.-W., \& Anthony, K. (2019b). Impact of window views on recovery-an example of post-cesarean section women. International Journal for Quality in Health Care: Journal of the International Society for Quality in Health Care, 31(10) 798-803. https://doi.org/10.1093/intqhc/mzz046

Watkins, N., Kennedy, M., Ducharme, M., \& Padula, C. (2011) Same-handed and mirrored unit configurations: Is there a difference in patient and nurse outcomes? Journal of Nursing Administration, 41(6), https://doi.org/10.1097/NNA.0b013e31821c47b4

Welton, J. M., Decker, M., Adam, J., \& Zone-Smith, L. (2006). How far do nurses walk? Medsurg Nursing, 15(4).

Yi, L., \& Seo, H.-B. (2012). The effect of hospital unit layout on nurse walking behavior. HERD: Health Environments Research \& Design Journal, 6(1), 66-82.

Zadeh, R. S., Shepley, M. M., Williams, G., \& Chung, S. S. E. (2014). The impact of windows and daylight on acute-care nurses' physiological, psychological, and behavioral health. HERD: Health Environments Research \& Design Journal, 7(4), 35-61.

Zimring, C., \& Seo, H.-B. (2012). Making acuity-adaptable units work: Lessons from the field. HERD: Health Environments Research \& Design Journal, 5(3), 115-128. 